\title{
The Born-Oppenheimer approximation in an effective field theory framework
}

\author{
Gastão Krein* \\ Instituto de Física Teórica, Universidade Estadual Paulista \\ Rua Dr. Bento Teobaldo Ferraz, 271 - Bloco II, 01140-070 São Paulo, SP, Brazil \\ E-mail: gastao.kreineunesp.br
}

\begin{abstract}
The Born-Oppenheimer approximation is the standard method for the studying atoms and molecules. It is founded on the observation that the energy scale of the electron dynamics in a molecule is larger than that of the nuclei. A very similar physical picture can be used to describe QCD states containing heavy quarks as well as light quarks and gluonic excitations. In this communication I present selected results of a recent work [N. Brambilla, G. Krein, J. Tarrús-Castellà and A. Vairo, Phys. Rev. D 97, 016016 (2018)] in which the Born-Oppenheimer approximation for atomic and hadronic molecular systems emerges as the leading-order approximation of an effective field theory obtained by sequentially integrating out degrees of freedom living at energies above the typical energy scale where the dynamics of the heavy degrees of freedom occurs. As an example, the simple case of a ion molecule formed by two heavy nuclei and one electron is considered.
\end{abstract}

XIII Quark Confinement and the Hadron Spectrum - Confinement2018

31 July - 6 August 2018

Maynooth University, Ireland

${ }^{*}$ Speaker. 


\section{Introduction and Motivation}

The Born-Oppenheimer approximation (BOA) [1] has been introduced in hadron physics more than thirty years ago [2, 3, 4, 5, 6, 7]. More recently, it has been suggested [8] that such an approximation could provide a good starting point for a coherent description of the so-called X,Y,Z exotic hadrons. These are hadrons observed at energies above the open charm (and bottom) production threshold. The underlying QCD dynamics governing their internal structure is yet to understood-for reviews, see Refs. $[9,10,11,12]$. The rational for the use of the BOA in this context is that the time scale associated with the dynamics of gluons and light quarks is small compared to that of the heavy quarks. In fact, the BOA has been used to study heavy hybrids by means of quenched lattice data for gluonic static potentials [13, 14, 15], but the new perspective advocated in Ref. [8]— see also Refs. [16, 17] — is that the BOA can also be applied to hadrons with light quarks when input from lattice simulations becomes available. In the present communication I will present selected results from Ref. [18], in which one step further in this proposal is taken and an effective field theory (EFT) is developed for calculating, in a systematic and controlled manner, corrections to the BOA for QED and QCD molecular systems.

The commonly used strategy in the construction of an EFT is the sequential integration of degrees of freedom induced by energy scales higher than the energy scale of the phenomenon of interest. In view of the lack of space, I will concentrate on the part of Ref. [18] related to the construction of an EFT for a QED molecule, more specifically, for a molecule formed by two heavy nuclei and one electron. For such a molecule, the sequential process of integrating out highenergy degrees of freedom proceeds as follows. First, hard modes associated with the masses of the charged particles are integrated out; this leads to the well known EFT of nonrelativistic QED (NRQED) $[19,20]$. Next, soft modes associated with the relative momenta of electrons and nuclei in NRQED are integrated out; this leads to the EFT known as potential NRQED (pNRQED) [21, 22]. Finally, the ultrasoft modes, associated with the electron and photon dynamics at the electron binding energy scale, are integrated out; this is done exploiting the fact the nuclei move much slower than the electrons. This results in an EFT for the motion of the nuclei only. In particular, the BOA emerges as the leading-order approximation in this EFT and as such it is named BornOppenheimer EFT (BOEFT). Because the interactions are weak, all these steps can be performed using perturbation theory.

\section{PNRQED}

The starting point of BOEFT is $\mathrm{PNRQED}$, an EFT suitable for describing QED at the ultrasoft scale. This EFT for the hydrogen atom was worked out in Ref. [22]. For molecules composed by two nuclei and one electron, it was worked out for the first time in Ref. [18]. To be specific, that reference considered a molecule formed by two heavy particles (nuclei) with electric charge $+Z e$ and mass $M$, and a light particle (electron) with electric charge $-e$ and mass $m$, with $M \gg m$. We recall that the relevant energy scales in such a QED bound state are the masses $M$ and $m$ (hard scales), the typical relative momentum $p=|\boldsymbol{p}| \sim m v$ between heavy and light particles (soft scale), and the binding energy of the light particles $E \sim m v^{2}$ (ultrasoft scale). For a Coulomb interaction, one still have $v \sim \alpha$ with $\alpha=e^{2} / 4 \pi \sim 1 / 137$. In addition, for this specific case an 
extra low-energy scale appears, namely the binding energy of the heavy nuclei. The operators of the pNRQED Lagrangian can be organized in an expansion in $\alpha$ and $m / M$. In order to homogenize the counting in these two expansion parameters, use is made of the fact that $m / M$ is numerically similar to $\sim \alpha^{3 / 2}$.

The Lagrangian of pNRQED can be written either in terms of the light and heavy fermion fields, or in terms of a composite-field operator representing the bound state. The process of obtaining the latter from the first is well documented in the literature, see e.g. Refs. [21, 22, 23]. For the specific case of the ion-molecule, this process was performed in great detail in Ref. [18]. Because of the lack of space, this will not be repeated here and we simply quote the final result. Before this, let us first state the center of mass (c.m.) and relative coordinates used:

$$
\boldsymbol{R}=\frac{m \boldsymbol{x}+M\left(\boldsymbol{y}_{1}+\boldsymbol{y}_{2}\right)}{m+2 M}, \quad \boldsymbol{z}=\boldsymbol{x}-\frac{\boldsymbol{y}_{1}+\boldsymbol{y}_{2}}{2}, \quad \boldsymbol{r}=\boldsymbol{y}_{1}-\boldsymbol{y}_{2},
$$

where $\boldsymbol{x}$ is the position vector of the electron and $\boldsymbol{y}_{1}$ and $\boldsymbol{y}_{2}$ the corresponding position vectors for the nuclei. The Lagrangian is given by [18]

$$
\begin{aligned}
L_{\mathrm{pNRQED}}= & -\frac{1}{4} \int d^{3} x F_{\mu v}(x) F^{\mu v}(x)+\int d^{3} R d^{3} r d^{3} z S^{\dagger}(t, \boldsymbol{R}, \boldsymbol{r}, \boldsymbol{z})\left[i \partial_{t}+\frac{\nabla_{R}^{2}}{2 M_{\mathrm{tot}}}+e_{\mathrm{tot}} A_{0}(t, \boldsymbol{R})\right. \\
& +e_{\mathrm{eff}} \boldsymbol{z} \cdot \boldsymbol{E}(t, \boldsymbol{R})-h_{0}(\boldsymbol{r}, \boldsymbol{z})+\frac{\boldsymbol{\nabla}_{r}^{2}}{M}-V_{\mathrm{ZZ}}^{\mathrm{LO}}(\boldsymbol{r})+\frac{\nabla_{z}^{2}}{4 M}+\frac{\boldsymbol{\nabla}_{z}^{4}}{8 m^{3}} \\
& \left.-V_{\mathrm{Ze}}^{\mathrm{NLO}}(\boldsymbol{z}+\boldsymbol{r} / 2, \boldsymbol{\sigma})-V_{\mathrm{Ze}}^{\mathrm{NLO}}(\boldsymbol{z}-\boldsymbol{r} / 2, \boldsymbol{\sigma})\right] S(t, \boldsymbol{R}, \boldsymbol{r}, \boldsymbol{z}),
\end{aligned}
$$

where $S(t, \boldsymbol{R}, \boldsymbol{r}, \boldsymbol{z})$ is the field operator representing the molecule, $F_{\mu \nu}=\partial_{\mu} A_{v}-\partial_{v} A_{\mu}$, with $A_{\mu}(t, \boldsymbol{x})$ representing ultrasoft photons (meaning that $A_{\mu}(t, \boldsymbol{x})$ must be multipole-expanded e.g. about the c.m. coordinate $\boldsymbol{R}), M_{\mathrm{tot}}$ is the total mass $M_{\mathrm{tot}}=m+2 M, \boldsymbol{E}(t, \boldsymbol{R})=-\partial_{t} \boldsymbol{A}(t, \boldsymbol{R})-\nabla_{R} A_{0}(t, \boldsymbol{R})$ is the electric field and $e_{\text {eff }}$ is the effective charge, $e_{\text {eff }}=2 e M+Z m / m+2 M=e+\mathscr{O}\left(\alpha^{2}\right)$. In addition,

$$
h_{0}(\boldsymbol{r}, \boldsymbol{z})=-\frac{\boldsymbol{\nabla}_{z}^{2}}{2 m}+V_{Z e}^{\mathrm{LO}}(\boldsymbol{z}+\boldsymbol{r} / 2)+V_{Z e}^{\mathrm{LO}}(\boldsymbol{z}-\boldsymbol{r} / 2),
$$

where $V_{Z e}(\boldsymbol{x}, \sigma)$ is the electron-nucleus potential, which contains contributions of order $m \alpha^{2}$ (leading order, LO) and $m \alpha^{4}$ (next-to-leading order, NLO):

$$
V_{Z e}(\boldsymbol{x}, \sigma)=V_{Z e}^{\mathrm{LO}}(\boldsymbol{x})+V_{Z e}^{\mathrm{NLO}}\left(\boldsymbol{x}, \sigma_{e}\right),
$$

with

$$
V_{Z e}^{\mathrm{LO}}(\boldsymbol{x})=-\frac{Z \alpha}{|\boldsymbol{x}|},
$$

being the ordinary Coulomb potential and

$$
V_{Z e}^{\mathrm{NLO}}\left(\boldsymbol{x}, \sigma_{e}\right)=-\frac{Z \alpha}{m^{2}}\left(-\frac{c_{D}}{8}+4 d_{2}\right) 4 \pi \delta(\boldsymbol{x})-i c_{S} \frac{Z \alpha}{4 m^{2}} \boldsymbol{\sigma}_{e} \cdot\left(\frac{\boldsymbol{x}}{|\boldsymbol{x}|^{3}} \times \boldsymbol{\nabla}_{x}\right),
$$

where the last term is a spin-orbit interaction, and $c_{D}, c_{S}$ and $d_{2}$ are matching coefficients, which up to order $\alpha$ are given by

$$
c_{D}=1+\frac{\alpha}{\pi}\left(\frac{8}{3} \log \frac{m}{\mu}\right), \quad c_{S}=1+\frac{\alpha}{\pi}, \quad d_{2}=\frac{\alpha}{60 \pi},
$$


with $\mu$ being the dimensional regularization scale. Finally, the $V_{Z Z}^{\mathrm{LO}}$ in Eq. (2.2) contains the LO nucleus-nucleus Coulomb potential:

$$
V_{Z Z}^{\mathrm{LO}}(\boldsymbol{x})=\frac{Z^{2} \alpha}{|\boldsymbol{x}|}
$$

Further contributions [24] to Eqs. (2.4) and (2.8) are beyond the desired accuracy. It is not difficult to show that the Lagrangian in Eq. (2.2) is invariant under the gauge transformation:

$$
A_{0}(t, \boldsymbol{R}) \rightarrow A_{0}(t, \boldsymbol{R})-\partial_{t} \theta(t, \boldsymbol{R}), \quad \boldsymbol{A}(t, \boldsymbol{R}) \rightarrow \boldsymbol{A}(t, \boldsymbol{R})+\nabla_{R} \theta(t, \boldsymbol{R}),
$$

under which the field $S(t, \boldsymbol{R}, \boldsymbol{r}, \boldsymbol{z})$ transforms as [18]

$$
S(t, \boldsymbol{R}, \boldsymbol{r}, z) \rightarrow e^{-i e_{\mathrm{tot}} \theta(t, \boldsymbol{R})} S(t, \boldsymbol{R}, \boldsymbol{r}, \boldsymbol{z})
$$

where $e_{\text {tot }}=-e(1-2 Z)$ is the total charge. Note that the field $S(t, \boldsymbol{R}, \boldsymbol{r}, z)$ is gauge invariant for a charge-neutral system, $e_{\mathrm{tot}}=0$.

\section{Born-Oppenheimer EFT: integrating out the ultrasoft modes}

Following the logic of EFTs, the ultrasoft degrees of freedom are to be integrated out from pNRQED to obtain an EFT at the energy scale of the two-nuclei dynamics, which is the BOEFT. This entails to integrating out the electron and ultrasoft photons. The three-level matching contributions can be obtained by expanding the field $S(t, r, z)$ in the pNRQED Lagrangian of Eq. (2.2) in eigenfunctions of the leading-order Hamiltonian $h_{0}(\boldsymbol{r}, \boldsymbol{z})$ of Eq. (2.3) (the c.m. coordinate $\boldsymbol{R}$ does not affect the internal dynamics of the molecule and therefore one can work in the c.m. frame, $\boldsymbol{R}=0)$ :

$$
S(t, \boldsymbol{r}, \boldsymbol{z})=\sum_{\kappa} \Psi_{\kappa}(t, \boldsymbol{r}) \phi_{\kappa}(\boldsymbol{r} ; \boldsymbol{z})
$$

where $\phi_{\kappa}(\boldsymbol{r} ; \boldsymbol{z})=\langle\boldsymbol{z} \mid \boldsymbol{r}, \kappa\rangle$ are the eigenstates of the electronic Hamiltonian

$$
h_{0}(\boldsymbol{r}, z) \phi_{\kappa}(\boldsymbol{r} ; z)=V_{\kappa}^{\text {light }}(\boldsymbol{r}) \phi_{\kappa}(\boldsymbol{r} ; z),
$$

where the eigenvalues $V_{K}^{\text {light }}(\boldsymbol{r})$ are the static energies and, for convenience, the eigenfunctions $\phi_{\kappa}(r ; z)$ taken to be orthonormal

$$
\int d^{3} z \phi_{\kappa}^{*}(\boldsymbol{r} ; z) \phi_{\kappa^{\prime}}(\boldsymbol{r} ; z)=\delta_{\kappa \kappa^{\prime}}
$$

Here, $\kappa$ represents the set of quantum numbers specifying the electronic state for a fixed separation $\boldsymbol{r}$ of the nuclei-in the usual notation employed in the field, a possible ground state is denoted by $\kappa={ }^{1} \Sigma_{g}^{+}$. Replacing the expansion in Eq. (3.1) into Eq. (2.2), one obtains

$$
\begin{aligned}
L_{\mathrm{BOEFT}}= & -\frac{1}{4} \int d^{3} x F_{\mu v}(x) F^{\mu v}(x)+\int d^{3} r \sum_{\kappa \kappa^{\prime}} \Psi_{\kappa}^{\dagger}(t, \boldsymbol{r})\left\{\left[i \partial_{t}+e_{\mathrm{tot}} A_{0}(t, \mathbf{0})-H_{\kappa}^{(0)}(\boldsymbol{r})\right.\right. \\
& \left.\left.-\delta E_{\kappa}(\boldsymbol{r})\right] \delta_{\kappa \kappa^{\prime}}-C_{\kappa \kappa^{\prime}}^{\mathrm{nad}}(\boldsymbol{r})\right\} \Psi_{\kappa^{\prime}}(t, \boldsymbol{r})
\end{aligned}
$$


where the photon fields here carry energies and momenta of $\mathscr{O}\left(m \alpha^{11 / 4}\right)$ or smaller. The different pieces in this Lagrangian have well-defined sizes [18]. Specifically, the leading-order term, the nuclei-nuclei Hamiltonian

$$
H_{\kappa}^{(0)}(\boldsymbol{r})=-\frac{\nabla_{r}^{2}}{M}+V_{Z Z}^{\mathrm{LO}}(\boldsymbol{r})+V_{\kappa}^{\mathrm{light}}(\boldsymbol{r}),
$$

has eigenvalues that scale as $m \alpha^{2}(m / M)^{1 / 2} \sim m \alpha^{11 / 4}$. The term $\delta E_{\kappa}(\boldsymbol{r})$ is the sum of the tree-level and second order recoil, Breit-Pauli and one-loop ultrasoft contributions:

$$
\delta E_{\kappa}(\boldsymbol{r})=\delta^{\mathrm{rec}} E_{\kappa}(\boldsymbol{r})+\delta^{\mathrm{rec}, 2} E_{\kappa}(\boldsymbol{r})+\delta^{\mathrm{NLO}} E_{\kappa}(\boldsymbol{r})+\delta^{\mathrm{US}} E_{\kappa}(\boldsymbol{r}),
$$

where

$$
\delta^{\mathrm{rec}} E_{\kappa}(\boldsymbol{r})=\left\langle\boldsymbol{r}, \kappa\left|\left(-\nabla_{z}^{2}\right) /(4 M)\right| \boldsymbol{r}, \kappa\right\rangle
$$

is of order $m \alpha^{7 / 2}$,

$$
\delta^{\mathrm{rec}, 2} E_{\kappa}(\boldsymbol{r})=\sum_{\bar{\kappa} \neq \kappa} \frac{\left|\left\langle\boldsymbol{r}, \kappa\left|\left(-\nabla_{z}^{2}\right) /(4 M)\right| \boldsymbol{r}, \bar{\kappa}\right\rangle\right|^{2}}{V_{\kappa}^{\text {light }}(\boldsymbol{r})-V_{\bar{\kappa}}^{\text {light }}(\boldsymbol{r})}
$$

is of order $m \alpha^{7 / 2}$

$$
\delta^{\mathrm{NLO}} E_{\kappa}(\boldsymbol{r})=\left\langle\boldsymbol{r}, \kappa\left|\left[V_{Z e}^{\mathrm{NLO}}(\boldsymbol{z}+\boldsymbol{r} / 2, \sigma)+V_{Z e}^{\mathrm{NLO}}(\boldsymbol{z}-\boldsymbol{r} / 2, \sigma)-\nabla_{z}^{4} / 8 m^{3}\right]\right| \boldsymbol{r}, \boldsymbol{\kappa}\right\rangle,
$$

is of order $m \alpha^{4}$,

$$
\begin{aligned}
\delta^{\mathrm{US}} E_{\kappa}(\boldsymbol{r})= & -\frac{e^{2}}{6 \pi^{2}}\left\{-\frac{Z e^{2}}{2 m^{2}}[\log (\mu / m)+5 / 6-\log (2)]\left[\left|\phi_{\kappa}(\boldsymbol{r} ; \boldsymbol{z}=\boldsymbol{r} / 2)\right|^{2}+\left|\phi_{\kappa}(\boldsymbol{r} ; \boldsymbol{z}=-\boldsymbol{r} / 2)\right|^{2}\right]\right. \\
& +\sum_{\bar{\kappa} \neq \kappa}\left|\left\langle\boldsymbol{r}, \kappa\left|\left(-i \nabla_{z} / m\right)\right| \boldsymbol{r}, \bar{\kappa}\right\rangle\right|^{2}\left(V_{\kappa}^{\text {light }}(\boldsymbol{r})-V_{\bar{\kappa}}^{\text {light }}(\boldsymbol{r})\right) \\
& \left.\times \log \left(m /\left|V_{\kappa}^{\text {light }}(\boldsymbol{r})-V_{\bar{\kappa}}^{\text {light }}(\boldsymbol{r})\right|\right)\right\},
\end{aligned}
$$

is of order $m \alpha^{5} \log \alpha$ and $m \alpha^{5}$ - the derivation of this latter result appears in the Appendix of Ref. [18]. In the above, we have used the short-hand notation

$$
\left.\langle\boldsymbol{r}, \kappa|\hat{O}(\boldsymbol{z}, \boldsymbol{r})| \boldsymbol{r}, \kappa\rangle=\int d^{3} z \phi_{\kappa}^{*}(\boldsymbol{r} ; \boldsymbol{z}) \hat{O}(\boldsymbol{z}, \boldsymbol{r}) \phi_{\kappa}(\boldsymbol{r} ; \boldsymbol{z}), 2\right)\left.\right|^{2} .
$$

We note that the $\overline{\mathrm{MS}}$ scheme has been used in the derivation of $\delta^{\mathrm{US}} E_{\kappa}(\boldsymbol{r})$. In addition, the $\mu$ dependence there cancels against that one of $c_{D}$ that enters the NLO potential in $\delta^{\mathrm{NLO}} E_{\kappa}(\boldsymbol{r})$. Finally, the term $C_{\kappa K^{\prime}}^{\mathrm{nad}}(\boldsymbol{r})$ is the nonadiabatic coupling [25]:

$$
C_{\kappa K^{\prime}}^{\mathrm{nad}}(\boldsymbol{r})=\int d^{3} z \phi_{\kappa}^{*}(\boldsymbol{r} ; z)\left[-\nabla_{r}^{2} / M \phi_{\mathcal{K}^{\prime}}(\boldsymbol{r} ; \boldsymbol{z})+2 / M\left(-i \nabla_{r} \phi_{\mathcal{K}^{\prime}}(\boldsymbol{r} ; \boldsymbol{z})\right)\left(-i \nabla_{r}\right)\right],
$$

where the first term is of order $m \alpha^{7 / 2}$ and the second is is of order $m \alpha^{25 / 8}$.

The Euler-Lagrange equation of this EFT provides a Schrödinger equation for the molecular energy levels. At leading order, when the nonadiabatic coupling can be neglected, the equation of motion for the field $\Psi_{\kappa}(t, r)$ is given by

$$
i \partial_{t} \Psi_{\kappa}(t, \boldsymbol{r})=H_{\kappa}^{(0)}(\boldsymbol{r}) \Psi_{\kappa}(t, \boldsymbol{r}) .
$$


This equation is nothing else than the Schrödinger equation that describes the motion of the heavy particles in the Born-Oppenheimer approximation [1]. On the other hand, the approximation known [25] as the adiabatic approximation corresponds to including the diagonal term $C_{\mathcal{K} K}^{\mathrm{nad}}(\boldsymbol{r})$ :

$$
i \partial_{t} \Psi_{\kappa}(t, \boldsymbol{r})=\left[H_{\kappa}^{(0)}(\boldsymbol{r})+C_{\kappa \kappa}^{\mathrm{nad}}(\boldsymbol{r})\right] \Psi_{\kappa}(t, \boldsymbol{r})
$$

I conclude here remarking that the BOEFT constructed in Ref. [18] is new, although NRQED has been used for nearly two decades $[20,26]$ to study atoms and molecules. The new and distinctive aspect in the construction of the BOEFT is that the full EFT program for the diatomic molecule is carried out. Namely, not only the hard scale is integrated out, as in NRQED, but also the soft and ultrasoft scales. The fact that the nuclei move slower than the electrons in a molecule, which is at the heart of the Born-Oppenheimer approximation, is equivalent to take the kinetic term of the nuclei to be of a smaller size than the energy scale of the electron dynamics, the ultrasoft scale. As these two scales are well separated, it is natural to integrate out the ultrasoft degrees of freedom. This leads to an EFT that describes the molecular degrees of freedom only. For accuracy at the level $\mathscr{O}\left(m \alpha^{4}\right)$, it is sufficient to match pNRQED and BOEFT at tree level. This matching is equivalent to expanding the molecular field in the pNRQED Lagrangian in eigenfunctions of the leading-order Hamiltonian for the electron. Loop diagrams involving ultrasoft photons, which are responsible the molecular Lamb shift, start contributing at $\mathscr{O}\left(m \alpha^{5}\right)$. The advantage of BOEFT is that each term in its Lagrangian has a unique size, a feature that is particularly useful for higher-order calculations as it greatly facilitates the determination of all the relevant contributions. All this was carried out explicitly and discussed throughly in Ref. [18].

\section{Conclusions and perspectives}

The BOEFT developed in Ref. [18] can be extended in a traightforward manner to QCD molecules, in particular to those composed of two heavy quarks bound adiabatically with some light degrees of freedom. Doubly-heavy baryons, which were first observed last year at the LHC [27], provide a primary example of such a possibility - this system was studied in the framework of pNRQCD in Ref. [28]. Pentaquarks [29], hadrons formed by two heavy quarks and three lightquarks, provide another possibility for the use of the BOEFT. This might be of particular relevance for the study of low-energy interactions of heavy quarkonia with nucleons [30] and also nuclearbound quarkonia, as such systems offer the opportunity to learn about the mass distribution inside a nucleon [31].

There is, however, a big difference between QED molecules and such QCD molecules, in that they are determined by nonperturbative interactions. Similarly to the QED molecule discussed here, the heavy quarks can be integrated out, as they move slower than the light degrees of freedom, whose dynamics appears at the scale $\Lambda_{\mathrm{QCD}}$, and an EFT for these QCD molecules can be constructed by integrating out the scale $\Lambda_{\mathrm{QCD}}$. But, since $\Lambda_{\mathrm{QCD}}$ is the scale of nonperturbative physics, the matching coefficients are nonperturbative quantities and need to be determined from models or, when they become available, from lattice calculations. When light quarks are neglected, one obtains an EFT recently constructed for quarkonium hybrids [32]—see also Ref. [33] and the contribution of Joan Soto to these proceedings. 


\section{References}

[1] R. Born and R. Oppenheimer, Zur Quantentheorie der Molekeln, Ann. Phys. 389 (1927) 457.

[2] P. Hasenfratz and J. Kuti, The Quark Bag Model, Phys. Rept. 40 (1978) 75.

[3] D. Horn and J. Mandula, A Model of Mesons with Constituent Gluons, Phys. Rev. D17 (1978) 898.

[4] P. Hasenfratz, R. R. Horgan, J. Kuti and J. M. Richard, The Effects of Colored Glue in the QCD Motivated Bag of Heavy Quark - anti-Quark Systems, Phys. Lett. 95B (1980) 299.

[5] P. Hasenfratz, R. R. Horgan, J. Kuti and J. M. Richard, Heavy Baryon Spectroscopy in the QCD Bag Model, Phys. Lett. 94B (1980) 401.

[6] D. Robson, Hadron Hadron Interactions With Orthogonal Lattice Gauge Field Configurations. 2. Nucleon Nucleon System, Phys. Rev. D35 (1987) 1029.

[7] S. Fleck and J. M. Richard, Baryons with double charm, Prog. Theor. Phys. 82 (1989) 760.

[8] E. Braaten, C. Langmack and D. H. Smith, Born-Oppenheimer Approximation for the XYZ Mesons, Phys. Rev. D90 (2014) 014044 [1402.0438].

[9] N. Brambilla et al., QCD and Strongly Coupled Gauge Theories: Challenges and Perspectives, Eur. Phys. J. C74 (2014) 2981 [1404.3723].

[10] R. A. Briceno et al., Issues and Opportunities in Exotic Hadrons, Chin. Phys. C40 (2016) 042001 [1511.06779].

[11] R. F. Lebed, R. E. Mitchell and E. S. Swanson, Heavy-Quark QCD Exotica, Prog. Part. Nucl. Phys. 93 (2017) 143 [1610.04528].

[12] F.-K. Guo, C. Hanhart, U.-G. Meißner, Q. Wang, Q. Zhao and B.-S. Zou, Hadronic molecules, Rev. Mod. Phys. 90 (2018) 015004 [1705.00141].

[13] K. J. Juge, J. Kuti and C. J. Morningstar, Gluon excitations of the static quark potential and the hybrid quarkonium spectrum, Nucl. Phys. Proc. Suppl. 63 (1998) 326 [hep-lat/9709131].

[14] L. A. Griffiths, C. Michael and P. E. L. Rakow, Mesons With Excited Glue, Phys. Lett. B129 (1983) 351.

[15] C. A. Meyer and E. S. Swanson, Hybrid Mesons, Prog. Part. Nucl. Phys. 82 (2015) 21 [1502.07276].

[16] N. Brambilla, A. Vairo, A. Polosa and J. Soto, Round Table on Heavy Quarkonia and Exotic States, Nucl. Phys. Proc. Suppl. 185 (2008) 107.

[17] A. Vairo, Quarkonia: a theoretical frame, in 3rd International Workshop on Charm Physics (Charm 2009) Leimen, Germany, May 20-22, 2009, 2009, 0912 . 4422.

[18] N. Brambilla, G. a. Krein, J. Tarrús Castellà and A. Vairo, Born-Oppenheimer approximation in an effective field theory language, Phys. Rev. D97 (2018) 016016 [1707.09647].

[19] W. E. Caswell and G. P. Lepage, Effective Lagrangians for Bound State Problems in QED, QCD, and Other Field Theories, Phys. Lett. B167 (1986) 437.

[20] T. Kinoshita and M. Nio, Radiative corrections to the muonium hyperfine structure. 1. The alpha**2 (Z-alpha) correction, Phys. Rev. D53 (1996) 4909 [hep-ph/9512327].

[21] A. Pineda and J. Soto, Effective field theory for ultrasoft momenta in NRQCD and NRQED, Nucl. Phys. Proc. Suppl. 64 (1998) 428 [hep-ph/9707481]. 
[22] A. Pineda and J. Soto, The Lamb shift in dimensional regularization, Phys. Lett. B420 (1998) 391 [hep-ph/9711292].

[23] N. Brambilla, A. Pineda, J. Soto and A. Vairo, Effective field theories for heavy quarkonium, Rev. Mod. Phys. 77 (2005) 1423 [hep-ph/ 0410047 ].

[24] C. Peset and A. Pineda, The Lamb shift in muonic hydrogen and the proton radius from effective field theories, Eur. Phys. J. A51 (2015) 156 [1508.01948].

[25] D. Marx and J. Hutter, Ab Initio Molecular Dynamics: Basic Theory and Advanced Methods. Cambridge University Press, 2009.

[26] P. Labelle, Effective field theories for QED bound states: Extending nonrelativistic QED to study retardation effects, Phys. Rev. D58 (1998) 093013 [hep-ph/9608491].

[27] LHCB collaboration, R. Aaij et al., Observation of the doubly charmed baryon $\Xi_{c c}^{++}, 1707.01621$.

[28] Brambilla, Nora and Vairo, Antonio and Rösch, Thomas, Effective field theory Lagrangians for baryons with two and three heavy quarks, Phys. Rev. D72 (2005) 034021 [hep-ph/ 0506065 ].

[29] LHCB collaboration, R. Aaij et al., Observation of J/ $\psi$ p Resonances Consistent with Pentaquark States in $\Lambda_{b}^{0} \rightarrow J / \psi K^{-}$p Decays, Phys. Rev. Lett. 115 (2015) 072001 [1507.03414].

[30] J. Tarrús Castellà and G. Krein, Effective field theory for the nucleon-quarkonium interaction, Phys. Rev. D98 (2018) 014029 [1803.05412].

[31] G. Krein, A. W. Thomas and K. Tsushima, Nuclear-bound quarkonia and heavy-flavor hadrons, Prog. Part. Nucl. Phys. 100 (2018) 161 [1706.02688].

[32] M. Berwein, N. Brambilla, J. Tarrús Castellà and A. Vairo, Quarkonium Hybrids with Nonrelativistic Effective Field Theories, Phys. Rev. D92 (2015) 114019 [1510.04299].

[33] Rubén Oncala and Joan Soto, Heavy Quarkonium Hybrids: Spectrum, Decay and Mixing, Phys. Rev. D96 (2017) 014004 [1 702.0390 0].

\section{Acknowledgements:}

Special thanks to Nora Brambilla, Jaume Tarrús-Castellá and Antonio Vairo for the collaborative work. Work partially financed by by Conselho Nacional de Desenvolvimento Científico e Tecnológico (CNPq), Grant Nos. 305894/2009-9, 464898/2014-5 (INCT Física Nuclear e Aplicações), and Fundação de Amparo à Pesquisa do Estado de São Paulo (FAPESP), Grant No.2013/019070. A grant from the bilateral agreement between Bayerische Hochschulzentrum für Lateinamerika (BAYLAT) of the Bayerischen Staatsministeriums für Bildung und Kultus, Wissenschaft und Kunst (StMBW) and Fundação de Amparo à Pesquisa do Estado de São Paulo (FAPESP), Contracts Nos. 914-20.1.3 (BAYLAT) and 2013/50841-1 (FAPESP) is acknowledged. The author still acknowledges a grant from the Bavarian State Ministry of Education, Science and the Arts through the TUM International Center Visiting Program 2016. 